Check for updates

Cite this: RSC Adv., 2018, 8, 12173

Received 14th February 2018

Accepted 15th March 2018

DOI: $10.1039 / c 8 r a 01442 f$

rsc.li/rsc-advances

\title{
Preparation of multi-layer nylon-6 nanofibrous membranes by electrospinning and hot pressing methods for dye filtration
}

\author{
Yuxi Yu, (D) *a Rui Ma, ${ }^{a}$ Shaole Yan ${ }^{a}$ and Jiyu Fang (D) *b
}

We report the formation of multi-layer nylon-6 (PA-6) nanofibrous membranes by electrostatic spinning coupled with a hot pressing process. The structure and porosity of multi-layer PA-6 nanofibrous membranes were characterized using a scanning electron microscope and the $\mathrm{N}_{2}$ nitrogen adsorption and desorption isotherms. We show that multi-layer PA-6 nanofibrous membranes can be used for efficient and continuous indigo dye filtration. Under the condition of constant pressure at the $0.1 \mathrm{MPa}$ dead end filtration, continuous filter for a period of time, the filtration efficiency for indigo dye increases with the increase of the number of layers in PA- 6 nanofibrous membranes. The 10 layer PA- 6 nanofiber membrane can completely remove the indigo dye, and early filtration flux was high, with extended time, the filtration flux decline and gradually stabilized.

\section{Introduction}

Nowadays, increasing attention has been devoted to polymer nanofibers because of their applications in filtration, fuel cells, sensors, drug delivery and tissue engineering. ${ }^{1-5}$ Electrospinning is a process in which a polymer fluid is drawn under electrostatic forces to form solid polymer nanofibers. ${ }^{6}$ Compared with various fiber-making routes including meltblown, ${ }^{7}$ template synthesis, ${ }^{8}$ sea-island spinning, ${ }^{9}$ phase-separation, ${ }^{10}$ and plasma treatment, ${ }^{11}$ electrospinning is a versatile and effective method for the large-scale fabrication of polymer nanofibers with controlled sizes, morphologies and functional components. ${ }^{12-14}$ Electrospun polymer nanofibrous membranes are a promising filtration material because of their high surface area to volume ratios, large porosity, good mechanical properties and good water permeability ${ }^{15}$ which are critical for air filtration ${ }^{16}$ and water treatment. ${ }^{17}$

Electrospun nylon-6 nanofibrous membranes as filtration materials have been reported in the literature. ${ }^{18-23}$ In this paper, we form multi-layer nylon-6 (PA-6) nanofibrous membranes by combing electrospinning and hot-pressing processes. The structure and porosity of multi-layer PA-6 nanofibrous membranes were characterized by a scanning electron microscope (SEM) and the $\mathrm{N}_{2}$ nitrogen adsorption and desorption isotherms. Furthermore, we study the filtration performance of multi-layer PA-6 nanofibrous membranes for indigo dye.

${ }^{a}$ Department of Materials Science and Engineering, College of Materials, Fujian Key Laboratory of Advanced Materials, Xiamen University, Xiamen, 361005, China. E-mail: yu_heart@xmu.edu.cn

${ }^{b}$ Department of Materials Science and Engineering and Advanced Materials Processing and Analysis Center, University of Central Florida, Orlando, Florida, 32816, USA

\section{Experimental section}

\section{Materials}

The nylon 6(PA-6 medium viscosity) was purchased from Toray industries, Inc., Japan. Formic acid (HCOOH, FA) and glacial acetic acid $\left(\mathrm{CH}_{3} \mathrm{COOH}, \mathrm{HAc}\right)$ were purchased from XiLong Scientific Co., Ltd, China. Indigo dye was provided by Brothers Chemical Co., Ltd. These chemicals were used without further purification.

\section{Preparation of PA-6 solution}

PA-6 was dissolved in FA/HAc mixed solution with the weight ratio of 3/1, in which the concentration of PA- 6 was $9 \mathrm{wt} \%$. The mixed solution was magnetically stirred at room temperature for 10-12 hours until it became a clear homogeneous solution.

\section{Fabrication of PA-6 nanofibrous membranes by electrospinning}

PA-6 solution was electrospun onto an aluminum foil support located $10 \mathrm{~cm}$ away from the edge of the cylinder electrode at ambient temperature. During electrospinning, a positive DC high voltage of $18 \mathrm{kV}$ was applied to PA-6 solution through the needle by using a high voltage power supply. In the electrospinning process, we need to maintain the speed of drum and the electrode to ensure the uniformity and continuity of nanofiber membranes.

\section{Preparation of multi-layer nanofibrous membranes and evaluation of filtration performance}

Fig. 1 shows the schematic diagram of multi-layer nanofibrous membranes and a filtration cell equipment. In order to 


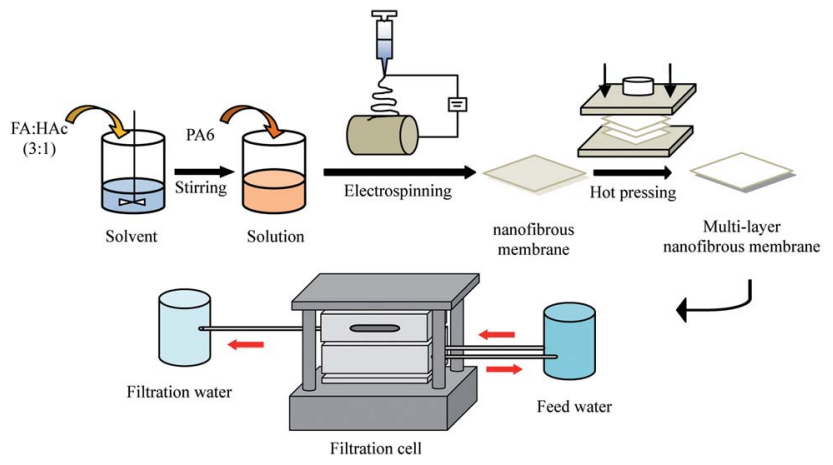

Fig. 1 Schematic diagram of the preparation of PA- 6 multi-layer nanofibrous membranes and filtration cell set-up.

completely remove the residual of solvents, the acquired nanofibrous membranes were placed in a vacuum oven at $60^{\circ} \mathrm{C}$ for $12 \mathrm{~h}$. Subsequently, 1, 2, 5 and 10 layers of electrospun PA-6 nanofibrous membranes were hot-pressed in flat-panel curing machine at $90{ }^{\circ} \mathrm{C}$ with different pressures for $120 \mathrm{~s}$. The principle of the filtration cell equipment was the dead end filter.

\section{Characterization}

The structure of electrospun PA-6 nanofibrous membranes was characterized with a scanning electron microscope (SEM, SU-70, Hitachi High-Tech, Tokyo, Japan) at $10 \mathrm{kV}$. For the SEM measurement, the membrane was placed on a conducting resin and then coated with gold. The diameters of fibers were measured and calculated using an image analyzer (Smile View). The surface area and pore size distribution of PA-6 nanofibrous membranes were analyzed by nitrogen gas adsorption and desorption method (TriStar II 3020, Micromeritics, USA). The nitrogen adsorption and desorption curves were measured at the temperature of liquid nitrogen $\left(-196{ }^{\circ} \mathrm{C}\right)$ using Micromeritics Tristar II 3020. Before the nitrogen adsorption, PA-6 nanofibrous membranes were degassed at $80{ }^{\circ} \mathrm{C}$ for $4 \mathrm{~h}$. The surface area and pore size distribution of PA- 6 nanofibrous membranes were obtained with Brunauer-Emmett-Teller (BET) and Barrett-Joyner-Halenda (BJH) methods, respectively. The UV-Vis spectra of filtrated dye solution of PA-6 nanofibrous membranes were taken with a UV-2550 UV-visible spectrophotometer (Shimadzu, Japan). The filtration flux of the fiber membrane was calculated according to the formula (1).

$$
J=\frac{V}{A \Delta t}
$$

In the formula, $J$ represents the filter flux, $\mathrm{L}\left(\mathrm{m}^{2} \mathrm{~h}\right)^{-1} ; V$ represents the body fluid through the nanofiber membrane, $\mathrm{L} ; A$ represents the effective filtration area, $\mathrm{m}^{2} ; t$ represents the time the liquid passed through the membrane, $h$.

\section{Results and discussion}

Fig. 2a is a photography image of PA-6 nanofibrous membranes. They are free-standing and flexible. The thickness of nanofibrous membranes increases with the increase of the number of layers (Fig. 2b). Fig. 3a-e shows the SEM image of PA-6 nanofibrous membranes. The electrospun PA-6 nanofibers have smooth surface and form three-dimensional networks. The diameter of the electrospun PA-6 nanofibers varies in the range from $0.1 \mu \mathrm{m}$ to $0.35 \mu \mathrm{m}$ (Fig. 3f).

Fig. 4 shows the $\mathrm{N}_{2}$ nitrogen adsorption and desorption isotherms of PA-6 nanofibrous membranes. The type-Iv isotherm evidenced by a hysteresis loop is a result of the porous structure of PA-6 nanofibrous membranes. A summary of the Brunauer-Emmett-Teller (BET) specific surface area and the average pore size of PA-6 nanofibrous membranes are given in Table 1. The average pore size of PA-6 nanofibrous membranes calculated with the Barrett-Joyner-Halenda (BJH) method slightly changes in the range from $8.54 \mathrm{~nm}$ to $12.15 \mathrm{~nm}$ with the increase of the number of layers in PA-6 nanofibrous membranes. The BET specific surface area decreases from 11.81 $\mathrm{m}^{2} \mathrm{~g}^{-1}$ to $5.68 \mathrm{~m}^{2} \mathrm{~g}^{-1}$ with the increase of the number of layers in PA-6 nanofibrous membranes.

Indigo dye is an anionic pollutant generated from industrial dyeing, printing and food coloring processes. The filtration
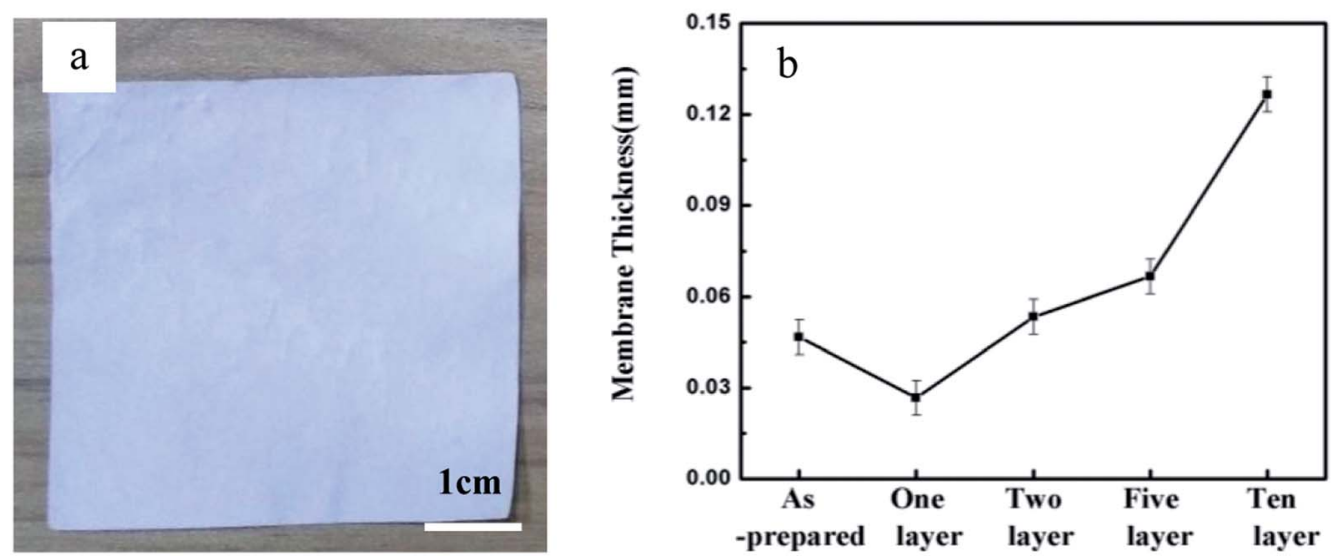

Fig. 2 (a) Photography image of 10 layer nanofibrous membranes. (b) The thickness of nanofibrous membranes as a function of the number of layers. 

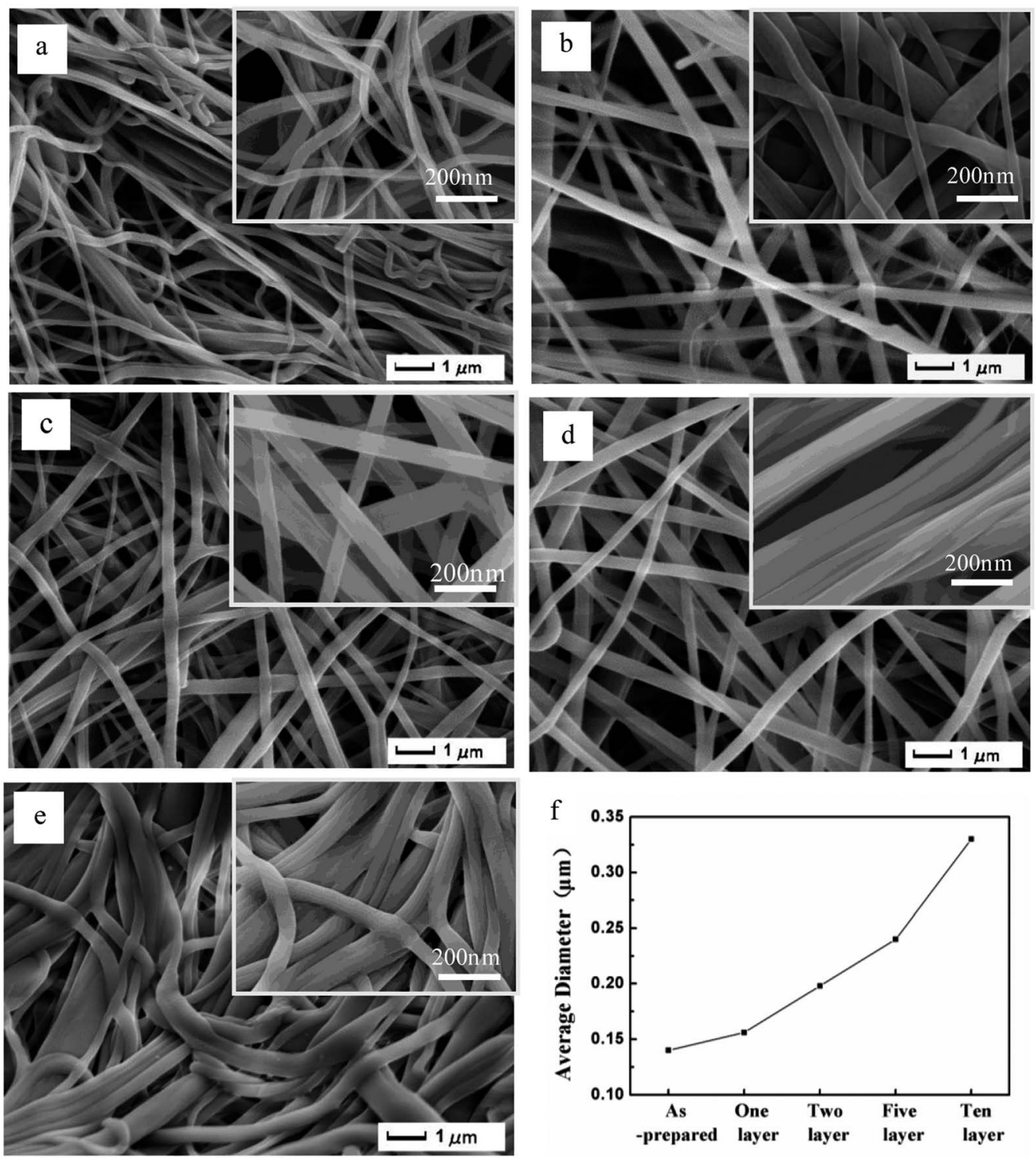

Fig. 3 SEM images of nanofibrous membrane with layer of (a) as-prepared (b) 1, (c) 2, (d) 5 and (e) 10. (f) Average fiber diameters of the relevant membranes.

performance of PA-6 nanofibrous membranes was carried out in a dead-end filtration cell equipment, in which indigo dye solution $\left(10 \mathrm{mg} \mathrm{L}^{-1}\right)$ was used as the feed solution. The dried nanofibrous membranes (the effective membrane area $\approx 0.02$ $\mathrm{m}^{2}$ ) was placed in the filtration cell and the feed solution was passed through the cell at the feed pressure of 0.1 MPa. The time required for the permeation of feed solution through the cell was recorded. Subsequently, the filtration measurements were repeated for three times.

Fig. 5 a shows the photography images of the top and bottom surfaces of 10-layer nanofibrous membranes after the filtration. After filtration, the top surface of fiber membrane surface was covered with a dense cake-layer of indigo dye. It can also be seen in Fig. 5b, indigo dye was trapped on the surface of the fibrous membrane, but the membrane was not completely blocked. This is because during the filtration process of the dead end, the filtrate was vertically passed through the membrane surface, and as the filtering time was performed, the concentration of the material deposited on the membrane surface produces a concentration polarization, so that the solute concentration of the membrane surface was greater than its saturation solubility, so that the filter cake was formed on the membrane surface adsorption.

Fig. 6 shows the UV-Vis absorption spectra of feed and filtration solution. The feed solution shows a maximum absorbance at $704 \mathrm{~nm}$. The intensity of the maximum absorbance was used to qualitatively evaluate the efficiency of PA-6 nanofibrous membranes for removing indigo dye. As can be seen in Fig. 6 that the intensity maximum absorbance at $704 \mathrm{~nm}$ of filtration solution decreases with the increase of the number of layers in nanofibrous membranes. The filtration efficiency of the one-layer membrane after the hot pressing is twice as high 


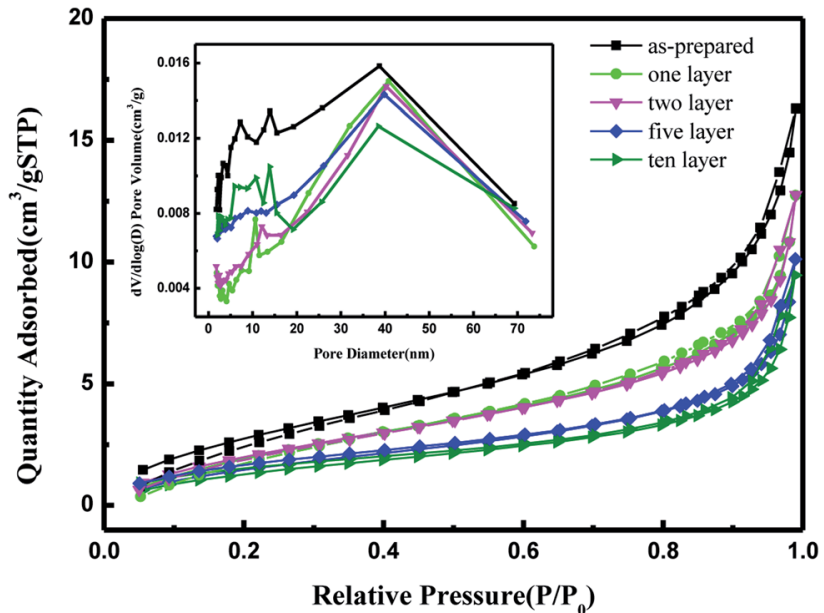

Fig. 4 Nitrogen adsorption-desorption isotherms and pore size distributions of PA- 6 nanofibrous membrane with different number of layers.

Table 1 Thickness, specific surface area, pore width of PA- 6 nanofibrous membranes

\begin{tabular}{llll}
\hline Sample & $\begin{array}{l}\text { Thickness } \\
(\mathrm{mm})\end{array}$ & $\begin{array}{l}\text { Specific surface } \\
\text { area }\left(\mathrm{m}^{2} \mathrm{~g}^{-1}\right)\end{array}$ & $\begin{array}{l}\text { Average pore } \\
\text { size }(\mathrm{nm})\end{array}$ \\
\hline As-prepared & 0.05 & 11.81 & 8.54 \\
One-layer & 0.03 & 9.80 & 9.13 \\
Two-layer & 0.05 & 9.18 & 9.63 \\
Five-layer & 0.07 & 6.53 & 11.25 \\
Ten-layer & 0.13 & 5.68 & 12.15 \\
\hline
\end{tabular}

as that of as-prepared membrane. After being filtrated by 10 layer nanofibrous membranes, the indigo dye are completely removed. In other words, the facile hot-pressing method proven to be very effective to improve the rejection fraction of electrospun nanofiber membranes. Fig. 7 shows the photography image of feed solution after filtration through as-prepared, 1, 2, 5 and 10 layer nanofibrous membranes. Fig. $7 \mathrm{~b}$ shows that blue color feed solution becomes clean and transparent after the filtration, which suggests that indigo dye molecules in solution are effectively removed by the membrane.

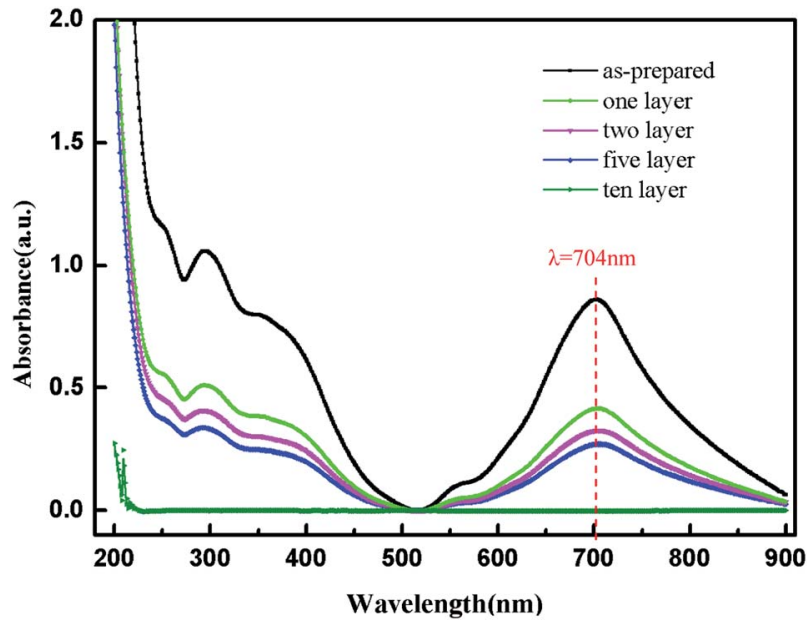

Fig. 6 UV-Vis absorption spectra of indigo dye solution before and after filtration by PA- 6 nanofibrous membranes with different layers.

Fig. 8 shows the water flux of electrospun PA-6 nanofiber membrane for removing indigo dye molecules in solution. Note that the 10 layer membrane was hot-pressed with $15 \mathrm{MPa}$ pressure. We can see that the filtration flux decreases rapidly in the first 200 minutes, then gradually slows down, and it remains above $15 \mathrm{~L} \mathrm{~m}^{-2} \mathrm{~h}^{-1}$ after 500 minutes of filtration, which is mainly the result of filter cake filtering. At first, under the pressure of a certain filtration pressure, most of the dye large particles were directly intercepted or adsorbed by the membrane and trapped on the membrane surface, and some small particles entered the membrane, so the initial filtration flux was large. With the continuous accumulation of the dye on the membrane surface and inside the membrane, a thick filter cake is formed on the film surface, the pore diameter of the film is reduced, the interception rate increases rapidly, and the filtration flux drops sharply. As the time goes on, the filter cake on the membrane tends to be stable, so the filtration flux of the membrane to the dye also tends to be stable. That is to say, when the thickness of cake layer reaches a certain value, the membrane have to be cleaned and remove the cake layer to restore the pre-filter state.
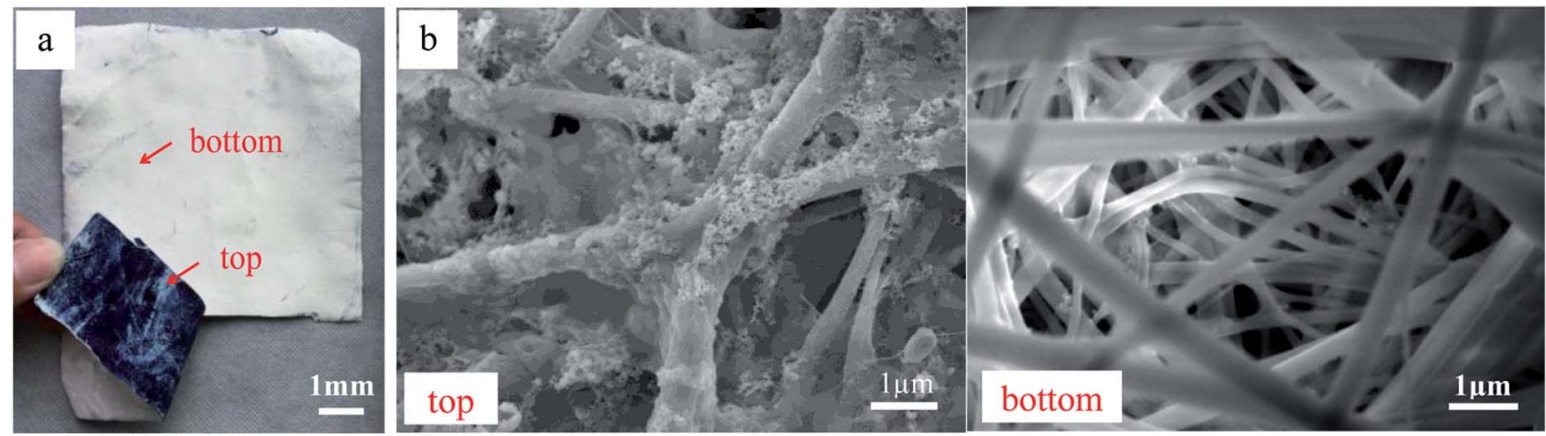

Fig. 5 (a) Photography images of the top and bottom surfaces of 10 layer nanofibrous membranes after filtration. (b) SEM image of 10 layer nanofibrous membranes after filtration. 


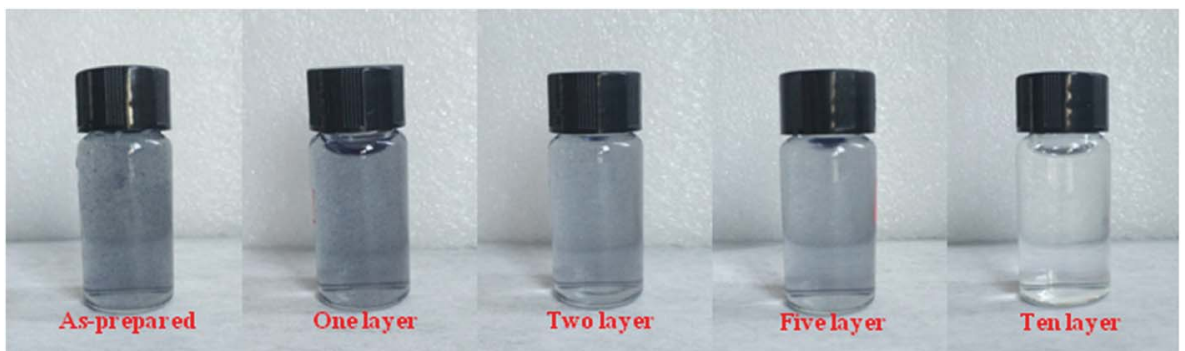

Fig. 7 Photography images of feed solution before (a) and after (b) filtration solution by PA- 6 nanofibrous membranes.

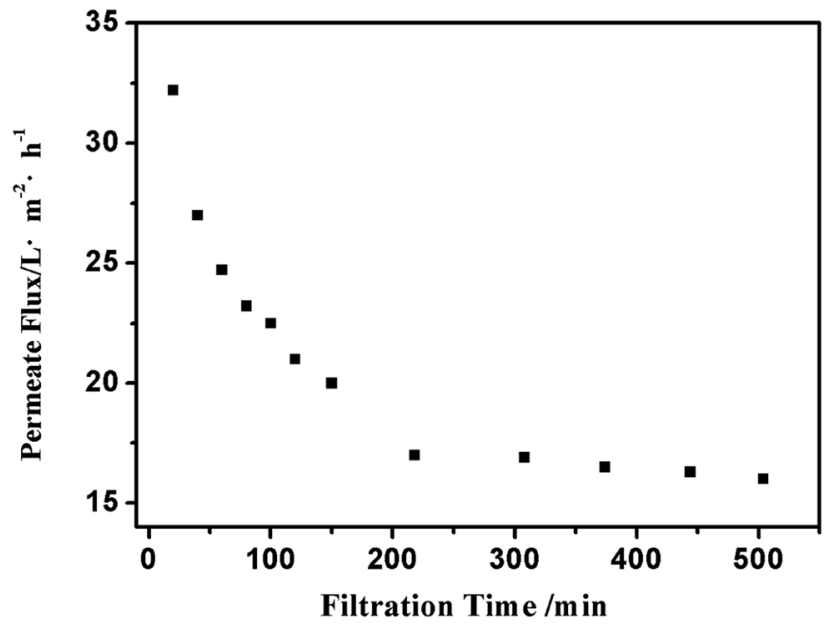

Fig. 8 Permeate flux of 10 layer nanofibrous membranes for removing indigo dye molecules in solution.

\section{Conclusions}

We have prepared multi-layer nanofibrous membranes by the hot-pressing of PA-6 nanofiber membranes prepared with electrostatic spinning. The structure and porosity of multi-layer PA-6 nanofibrous membranes were characterized by SEM and the $\mathrm{N}_{2}$ nitrogen adsorption and desorption isotherms. After PA6 nanofiber membrane was hot-pressing, with increasing number of layer, the fiber diameters and the thickness of membrane increased. We showed that multi-layer PA-6 nanofibrous membrane can be used as a filtration material to remove indigo dye from water, and it can be continuously filtered for a long time. The filtration efficiency for indigo dye increases with the increase of the number of layers in PA-6 nanofibrous membranes. The 10 layer PA-6 nanofiber membrane can completely remove the indigo dye, and early filtration flux was high, with extended time, the filtration flux decline and gradually stabilized. The ability to intercept dye molecules and in a simple one-step hot-pressing process will enhance their applicability significantly in the field of filtration.

\section{Conflicts of interest}

There are no conflicts to declare.

\section{Acknowledgements}

The financial support of this work was provided by the Natural Science Foundation of China (51675452), and the Research Foundation (6142A060403, 6140923020301) are acknowledged.

\section{References}

1 V. Thavasi, G. Singh and S. Ramakrishna, Electrospun nanofibers in energy and environmental applications, Energy Environ. Sci., 2008, 1(2), 205-221.

2 Q. P. Pham, U. Sharma and A. G. Mikos, Electrospinning of polymeric nanofibers for tissue engineering applications: a review, Tissue Eng., 2006, 12(5), 1197-1211.

3 B. Ding, $\mathrm{M}$. Wang, $\mathrm{X}$. Wang, et al. Electrospun nanomaterials for ultrasensitive sensors, Mater. Today, 2010, 13(11), 16-27.

4 V. Leung, R. Hartwell and E. Faure, et al. Electrospun nanofibers as next-generation bioactive tissue scaffolds, Tissue engineering and regenerative medicine. A Nano Approach, ed. M. Ramalingam, P. Vallittu, U. Ripamonti, W. J. Li, Taylor \& Francis Group, Florida, 2013, pp. 229-246.

$5 \mathrm{~V}$. Leung and F. Ko, Biomedical applications of nanofibers, Polym. Adv. Technol., 2011, 22(3), 350-365.

6 A. Greiner and J. H. Wendorff, Electrospinning: a fascinating method for the preparation of ultrathin fibers, Angew. Chem., Int. Ed., 2007, 46(30), 5670-5703.

7 K. Watanabe, Y. Suzuki, T. Goto, et al. Continuous hemodiafiltration in children after cardiac surgery, Artif. Organs, 2011, 35(3), 288-293.

8 P. Qiu and C. Mao, Biomimetic branched hollow fibers templated by self-assembled fibrous polyvinylpyrrolidone structures in aqueous solution, ACS Nano, 2010, 4(3), 15731579.

9 D. Wang, G. Sun and B. S. Chiou, A high throughput, controllable, and environmentally benign fabrication process of thermoplastic nanofibers, Macromol. Mater. Eng., 2007, 292(4), 407-414.

$10 \mathrm{~J}$. Mao, S. Duan, A. Song, et al. Macroporous and nanofibrous poly (lactide-co-glycolide)(50/50) scaffolds via phase separation combined with particle-leaching, Mater. Sci. Eng., C, 2012, 32(6), 1407-1414.

11 K. Mondal, M. A. Ali, V. V. Agrawal, et al. Highly sensitive biofunctionalized mesoporous electrospun $\mathrm{TiO}_{2}$ nanofiber 
based interface for biosensing, ACS Appl. Mater. Interfaces, 2014, 6(4), 2516-2527.

12 J. D. Schiffman and C. L. Schauer, A review: electrospinning of biopolymer nanofibers and their applications, Polym. Rev., 2008, 48(2), 317-352.

13 A. Cipitria, A. Skelton, T. R. Dargaville, et al. Design, fabrication and characterization of PCL electrospun scaffolds - a review, J. Mater. Chem., 2011, 21(26), 9419-9453.

14 M. Tian, Y. N. Wang and R. Wang, Synthesis and characterization of novel high-performance thin film nanocomposite (TFN) FO membranes with nanofibrous substrate reinforced by functionalized carbon nanotubes, Desalination, 2015, 370, 79-86.

15 C. Burger, B. S. Hsiao and B. Chu, Nanofibrous materials and their applications, Annu. Rev. Mater. Res., 2006, 36, 333-368.

16 K. Yoon, B. S. Hsiao and B. Chu, Functional nanofibers for environmental applications, J. Mater. Chem., 2008, 18(44), 5326-5334.

17 R. Wang, Y. Liu, B. Li, et al. Electrospun nanofibrous membranes for high flux microfiltration, J. Membr. Sci., 2012, 392, 167-174.
18 D. Aussawasathien, C. Teerawattananon and A. Vongachariya, Separation of micron to sub-micron particles from water: electrospun nylon-6 nanofibrous membranes as pre-filters, J. Membr. Sci., 2008, 315(1), 11-19.

19 H. R. Pant, Biomimetic spider-web like electrospun nanofibrous membrane of nylon- 6 for future air filtration, Journal of the Institute of Engineering, 2016, 11(1), 108-115.

20 F. E. Ahmed, B. S. Lalia and R. Hashaikeh, A review on electrospinning for membrane fabrication: challenges and applications, Desalination, 2015, 356, 15-30.

21 M. Aliabadi, Effect Of Electrospinning Parameters On The Air Filtration Performance Using Electrospun Polyamide-6 Nanofibers, Chem. Ind. Chem. Eng. Q., 2017, 23(4), 441-446.

22 H. Amir Houshang, R. Abosaeed, G. Reza, et al. Effect of needle length, electrospinning distance, and solution concentration on morphological properties of polyamide- 6 electrospun nanowebs, Text. Res. J., 2013, 83(14), 1452-1466. 23 Y. Guibo, Z. Qing, Z. Yahong, et al. The Electrospun Polyamide 6 Nanofiber Membranes Used as High Efficiency Filter Materials: Filtration Potential, Thermal Treatment, and Their Continuous Production, J. Appl. Polym. Sci., 2013, 128(2), 1061-1069. 